\title{
Ce que disent des élèves sur les classes coopératives en collège et lycée
}

\section{Sylvain Connac}

\section{(2) OpenEdition \\ Journals}

Édition électronique

URL : http://journals.openedition.org/trema/4265

DOI : $10.4000 /$ trema.4265

ISSN : 2107-0997

Éditeur

Faculté d'Éducation de l'université de Montpellier

Édition imprimée

Date de publication : 1 novembre 2018

ISSN : 1167-315X

Référence électronique

Sylvain Connac, " Ce que disent des élèves sur les classes coopératives en collège et lycée », Tréma [En ligne], 50 | 2018, mis en ligne le 01 novembre 2018, consulté le 19 avril 2019. URL : http:// journals.openedition.org/trema/4265 ; DOI : 10.4000/trema.4265

Ce document a été généré automatiquement le 19 avril 2019.

Trema 


\title{
Ce que disent des élèves sur les classes coopératives en collège et lycée
}

\author{
Sylvain Connac
}

\section{Introduction}

1 Cela fait longtemps que des projets de classes coopératives existent, principalement en écoles primaires. Elles sont le fruit de multiples travaux, à l'initiative de grands pédagogues comme G. Girard, J.H. Pestalozzi, J. Dewey, B. Profit, E. Bugnon, E. Claparède, R. Cousinet, E. et C. Freinet, F. Oury, R. Laffitte, P. Meirieu ou C. Héber-Suffrin. Depuis quelques temps, des classes coopératives se créent au sein d'établissements du second degré, à la fois pour motiver les élèves, prendre en compte leur diversité, améliorer le climat scolaire, accroitre le rapport au savoir, susciter la citoyenneté démocratique. Plusieurs invitations du Ministère de l'Education Nationale encouragent les équipes de professeurs à prendre cette voie. "La coopération entre élèves est une entrée qui promeut des élèves acteurs de leur scolarité, de leur citoyenneté et de leurs apprentissages. » (Florence Robine, in Veltcheff, C. Vincent, J. F., 2016, p. 3)

2 Les pratiques de la coopération scolaire défendent des valeurs comme la fraternité, la solidarité, le respect, l'engagement, l'ouverture aux autres, de droit à la différence, la confiance, le partage, le plaisir, l'autonomie, l'équité ou l'écoute empathique. (Howden, Rouiller, 2009, p. 20) La coopération est avant tout une organisation collective qui vise un intérêt général. Elle regroupe toutes les situations où enfants, jeunes et adultes, réunis en communautés de recherche, mettent à disposition de tous les richesses individuelles, échangent leurs connaissances et développent ainsi des habiletés métacognitives (Connac, 2009).

3 Dans une optique de rapport au savoir, quatre formes de relations coopératives entre pairs ont été isolées : l'aide, l'entraide, le travail en groupe et le tutorat (Connac, 2017a). Ces quatre déclinaisons s'attachent à déterminer des interactions coopératives en 
groupes restreints. Nous les complétons par des pratiques plus collectives, pensées comme des démarches de projets, présentes avec l'organisation de conseils coopératifs, de réseaux d'échanges réciproques de savoirs, de médiation entre pairs, de correspondance interclasses, de discussions à visées démocratiques et philosophiques, de démarches de projets ou de jeux coopératifs. Une classe coopérative correspond à une organisation pédagogique qui convoque tout ou partie de ces déclinaisons. En collèges ou lycées, elle nécessite conjointement une structuration de la collaboration entre adultes (enseignants disciplinaires et personnels de vie scolaire) qui forment une équipe pédagogique en charge d'une ou plusieurs classes coopératives.

Dans le cadre d'accompagnements par la recherche de classes coopératives en collèges et lycées d'établissements publics, nous avons pu observer les tâtonnements et les évolutions pédagogiques d'équipes pluridisciplinaires d'enseignants. Cette étude propose une analyse d'un corpus de 22 entretiens semi-directifs de recherche, avec des élèves de ces classes. Leur analyse permet des réponses à cette question de recherche : que disent des élèves en collèges et en lycées de ce qu'ils vivent au sein de classes dites coopératives? L'analyse de leurs discours permet d'accéder à la réalisation des projets pédagogiques conduits par les enseignants, d'en étudier la nature et d'en estimer la portée au regard des intentions posées. Les objectifs pédagogiques énoncés par les enseignants sont-ils perçus de la sorte par leurs élèves? Outre le fait que cette étude conduit à la parole des premiers concernés par la pédagogie, à savoir, les élèves, elle autorise des éléments de rétroaction utiles pour celles et ceux souhaitant, à leur tour, mettre en œuvre un projet de classe coopérative en collège ou lycée.

\section{Coopération entre élèves, quelques précisions sémantiques}

La coopération entre élèves regroupe l'ensemble des situations scolaires où des élèves sont amenés à apprendre ou à produire à plusieurs. Ils agissent ensemble, exercent de la fraternité et développent du désir (Go, in Sumputh, Fourcade, 2013). Des élèves peuvent coopérer de différentes manières : dans un lien de parité, on parle de relation symétrique ; lorsqu'un élève expert échange avec un camarade en demande, il s'agit plutôt de relation dissymétrique. Certaines formes de coopération sont formelles, avec une consigne à l'origine d'un enseignant, d'autres ont un caractère informel et se produisent à l'initiative des élèves.

La collaboration concerne principalement l'activité d'adultes. Elle décrit des situations de travail, alors que la coopération peut aussi traduire du jeu. Elle regroupe les pratiques coopératives symétriques, des collaborateurs recherchant de la solidarité (s'associer pour unir les forces) là où des coopérateurs peuvent aussi tendre vers de la générosité (réaliser un don gratuit de soi). Enfin, alors que collaborer serait participer à un projet commun, coopérer impliquerait un agir conjoint, dans un même espace au même moment (Marcel et al., 2007).

Les pratiques de coopération entre élèves n'englobent pas les interactions avec un enseignant, ni celles avec du matériel pédagogique. Elles se déclinent par des situations centrées sur les apprentissages (l'aide, l'entraide, le tutorat, le travail en groupe, le travail en équipe ou en atelier) et d'autres centrées sur l'entretien du collectif (les conseils coopératifs, les jeux coopératifs, les marchés de connaissances ...). (Connac, 2017)

\section{Différentes formes coopératives}

Une situation d'aide se produit lorsqu'un élève qui pense pouvoir aider, essaie 
d'apporter ses connaissances et ses compétences à un camarade qui en a exprimé le besoin. Le tutorat intervient suite à une formation préalable des élèves : un tuteur est celui qui tente de répondre à une question posée par un tutoré (Baudrit, 2017). Le tutorat regroupe l'activité des tuteurs et celle des tutorés, dans une logique de réciprocité où tous les élèves investissement alternativement ces deux fonctions. C'est cette dimension de réciprocité qui distingue tutorat de monitorat, tant un moniteur a besoin d'être un expert des savoirs (Oursel, 2016). Le tuteur n'est qu'un soutien possible.

L'entraide se produit lorsque deux ou plusieurs élèves prennent l'initiative de se réunir pour tenter, à plusieurs, de résoudre un problème ou une difficulté qu'ils rencontrent conjointement (Marchive, 1995). Le travail en groupe concerne plutôt une forme de mise au travail des élèves à l'initiative d'un enseignant. Il compte sur la confrontation des idées pour déclencher chez les élèves un litige cognitif afin qu'ils se posent des questions auxquelles les savoirs en jeu vont pouvoir apporter des éléments de réponse (Meirieu, 1996). Un travail en équipe concerne les formes de coopération relatives aux démarches de projets, principalement orientées vers les "éducations à". En s'y engageant à plusieurs, les élèves réinvestissent des apprentissages et développent des compétences transversales (mener un projet à terme, rédiger des comptes-rendus, prendre la parole en public ...). Un atelier est orienté vers l'usage d'un matériel spécifique ou un travail autour d'apprentissages précis. Des élèves sont conduits à rejoindre un même atelier pour développer des compétences socio-comportementales.

Un conseil coopératif d'élèves se veut une réunion démocratique qui cherche à harmoniser la vie du groupe en associant les participants : faire l'état des lieux des projets personnels ou collectifs, aborder les propositions, les problèmes, la coopérative, les félicitations mais aussi tout ce qui concerne le symbolique dans la classe (permis, brevets, ceintures, responsabilités, ...). Au sein d'une classe, un conseil coopératif est reconnu comme la clé de voute de l'exercice de l'autonomie des élèves (Le Gal, 2002).

Le principe d'un jeu coopératif est l'engagement physique ou intellectuel, intense mais sans gagnant ni perdant, sans compétition ni violence, dans le but de réaliser un défi ou de battre un record précédemment établi. Un marché de connaissances (autrement désigné par "réseau d'échanges réciproques de savoirs") réunit des élèves qui transmettent des savoirs à des receveurs (Héber-Suffrin et al., 2011). Le postulat de départ est que chacun possède et est capable de mettre à disposition des savoirs qui peuvent intéresser d'autres personnes. La réciprocité oblige les passeurs à devenir receveurs et inversement.

Il existe plusieurs autres formes d'organisations coopératives du travail des élèves, notamment les discussions à visées démocratique et philosophique qui aident à développer des habiletés de pensée réflexive (Tozzi, 2012). 
Figure 1 : Carte conceptuelle de la coopération entre élèves

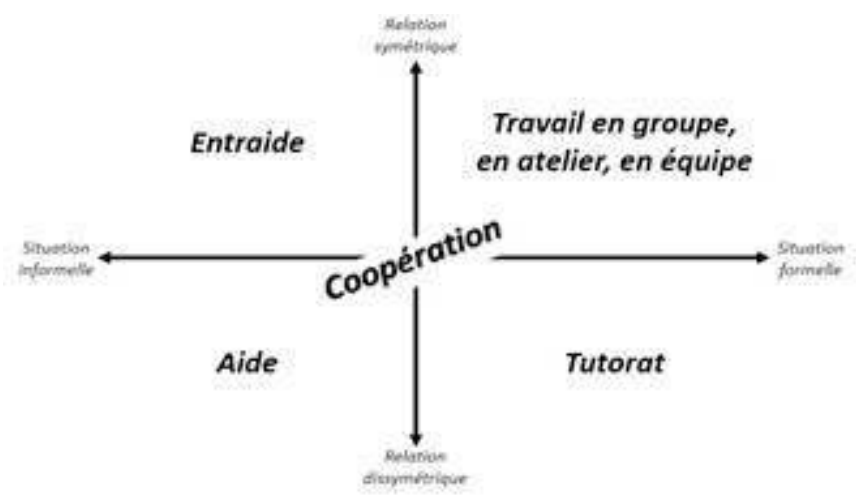

\section{Pédagogies et classes coopératives}

Les pédagogies coopératives regroupent toutes celles qui convoquent des formes variées de coopérations entre élèves, en complément de situations où ils sont amenés à travailler seul ou en relation avec un adulte : Pédagogies Freinet, Institutionnelle, Cousinet ... Elles font l'objet d'évolutions par l'intermédiaire de plusieurs mouvements pédagogiques (OCCE, ICEM, CRAP-Cahiers Pédagogiques ...). Une classe coopérative se constitue dès lors que les élèves sont amenés à coopérer. Elle correspond à des organisations diverses, allant de formes modestes, jusqu'à des structures globales, à l'image de ce qui se développe en Pédagogie Freinet (ICEM), en passant par l'entretien avec les enfants d'une coopérative scolaire, selon le projet éducatif de l'OCCE (Office Central de la Coopération à l'Ecole).

S'orienter vers une pédagogie coopérative en tant qu'enseignant, c'est poursuivre plusieurs finalités et participer à un projet d'éducation plus large que celui de la classe. Lorsque des élèves coopèrent, ils sont sensés affermir leur autonomie et leur responsabilité en même temps que leurs capacités sociales. En coopérant, l'intention est de sensibiliser les élèves à la force des collectifs, les groupes altruistes supplantant les groupes égoïstes (Servigne, Chapelle, 2017).

De plus, des élèves qui coopèrent se trouvent dans un contexte scolaire où les hétérogénéités sont considérées comme des sources d'enrichissements mutuels. C'est en ce sens, par la pluralité des formes possibles d'apprentissages, qu'une classe coopérative s'apparente à une démarche de différenciation pédagogique, permettant la prise en compte de la diversité des élèves par l'encouragement des comportements prosociaux.

\section{Pour aller plus loin}

Baudrit, A. (2007b). Le tutorat, richesse d'une méthode pédagogique. Bruxelles : De Boeck. Connac, S. (2017). La coopération entre élèves. Futuroscope : Editions Canopé.

Heber-Suffrin, C., Renon Thouret, C., Desgroppes, N., Vidricaire, A. (2011). Parier sur la réciprocité, vivre la solidarité. Lyon : Chronique Sociale.

Le Gal, J. (2002). Les droits de l'enfant à l'école, pour une éducation à la citoyenneté. Bruxelles : De Boeck et Belin.

Marcel, J.F., Dupriez, V., Périsset, D, Tardif, M. (2007). Coordonner, collaborer, coopérer : de nouvelles pratiques enseignantes. Bruxelles : De Boeck Universités.

Marchive, A. (1995). L'entraide entre élèves à l'école élémentaire. Relations d'aide et 
interactions pédagogiques entre pairs de six classes du cycle trois. Thèse de doctorat en sciences de l'éducation, Bordeaux : Université de Bordeaux II.

Meirieu, P. (1996). Itinéraire des pédagogies de groupe - Apprendre en groupe. Lyon :

Chronique Sociale.

Oursel, F. (2016). Du monitorat au tutorat. Education permanente, 206/2016-1, 11-18.

Servigne, P., Chapelle, G. (2017). L'entraide - L'autre loi de la jungle. Paris : Les liens qui libèrent.

Sumputh, M., Fourcade, F. (2013). Oser la pédagogie coopérative complexe. Lyon : Chronique Sociale.

Tozzi, M. (2012). Nouvelles pratiques philosophiques. Lyon : Chronique Sociale.

\section{Problématique et méthodologie}

L'intérêt d'écouter ce qu'expriment des élèves de classes coopératives est multiple. En effet, ce sont eux qui vivent au quotidien ce que les enseignants parviennent à organiser en matière de pratiques coopératives. Ils sont donc à même de témoigner de la nature et des effets de ces approches pédagogiques. D'autant plus, qu'en tant que collégiens ou lycées, leur scolarité passée les a conduits à rencontrer d'autres façons d'enseigner. Ils se présentent ainsi tels des révélateurs, apportant une distanciation quant à ce que des enseignants pourraient dire de ces mêmes réalités. N'étant pas à l'origine de ces projets et ne pouvant être accusés de quelconque idéologie, ils présentent un vécu ordinaire, tel que compris personnellement. De plus, écouter des élèves s'exprimer sur la façon dont ils travaillent permet d'entrer dans le grain fin du fonctionnement d'une classe coopérative. Ils proposent une visite détaillée, à partir de ce qu'ils estiment important et qui peut différer de ce que souligneraient des adultes. Cette recherche du détail des organisations coopératives est permise par le croisement des propos collectés par les entretiens. Chaque élève ne dit pas tout, mais la complémentarité de leurs discours présente une pluralité de fonctionnements de classe. Enfin, ce que disent les élèves est à l'image de leur posture en classe, on accède ainsi à une partie de ce que forment les projets de classes coopératives en matière de "métiers d'élèves » (Perrenoud, 2004), entendus ici comme des prédispositions mentales dans le rapport à la scolarisation. Cette recherche ne peut attester de ce que les élèves apprennent par la coopération mais elle peut donner matière à comprendre quels types de postures sont suscités. Par exemple, elle permet d'éprouver la réciprocité entre tuteurs et tutorés, afin que constater si les élèves se sont bien appropriés la logique d'alternance entre ces deux fonctions.

6 Cette approche se situe donc dans une logique phénoménologique, appliquée à des élèves qui vivent la scolarisation à travers ce que leurs enseignants développent. "La phénoménologie, c'est l'étude des essences, et tous les problèmes, selon elle, reviennent à définir des essences : l'essence de la perception, l'essence de la conscience, par exemple. Mais la phénoménologie, c'est aussi une philosophie qui replace les essences dans l'existence et ne pense pas qu'on puisse comprendre l'homme et le monde autrement qu'à partir de leur "facticité ». (Merleau-Ponty, 1945, p. I). On développe donc une approche de primarisation (Etienne, 2009) qui s'attache à la compréhension des faits par les élèves, sans recherche des intentions véhiculées par leurs enseignants. Contrairement à une observation participante, cette attention à la parole des élèves réduit la déformation 
induite par l'interprétation des faits par le chercheur, dans le sens où seul le discours des élèves sert à l'analyse. C'est cette unique base qui constitue la source des données.

7 Nous avons procédé à des entretiens semi directifs de recherche auprès d'élèves de classes coopératives. $(n=22)$ Trois établissements (11 classes) ont servi de support : un collège en éducation prioritaire $\left(12\right.$ entretiens d'élèves en $6^{\text {ème }}$ - codés A dans la recherche), un collège en milieu rural ( 1 élève en $6^{\text {ème }}$ et 4 élèves en $5^{\text {ème }}$ - codés $B$ ) et un lycée d'enseignement général et technologique ( 5 élèves en $2^{\text {nde }}$ - codés $C$ ). Dans le collège $\mathrm{A}$, les 7 sixièmes sont des classes coopératives. Dans le collège $\mathrm{B}$, les enseignants ont créé un parcours de la $6^{\text {ème }}$ à la $3^{\text {ème }}$, avec une classe coopérative dans chaque niveau. Dans le lycée $C$, deux secondes sont des classes coopératives. Au moment des entretiens, le collège A en est à sa cinquième année de vie de classes coopératives, le collège $\mathrm{B}$ à sa deuxième année et le lycée $\mathrm{C}$ à sa troisième.

8 Ces établissements publics ne sont pas des collèges et lycées expérimentaux. Ils se trouvent dans des territoires divers, accueillent les élèves de leurs bassins d'affectation et sont composés d'enseignants qui intègrent les équipes pédagogiques selon les barèmes du mouvement des professionnels. Ils ne disposent pas de moyens spécifiques pour faire vivre ces projets de classes coopératives. En revanche, ils ont demandé et obtenu une contractualisation avec la CARDIE (Cellule Académique de Recherche et Développement pour l'Innovation et l'Expérimentation) auprès de leurs services rectoraux et disposent d'un accompagnement par un conseiller en développement pour valoriser leur projet par la rédaction d'une fiche Expérithèque (la Bibliothèque Nationale des expérimentations pédagogiques).

9 Les enseignants ont mis en place ces classes coopératives pour des raisons variables selon les besoins des établissements. Le collège $\mathrm{A}$ tente ainsi de réagir face à un grave problème d'assiduité des élèves et propose d'y répondre par des pédagogies qui suscitent davantage leur intérêt et leur motivation. Le collège $\mathrm{B}$ est confronté à une grande hétérogénéité au sein des classes, tant au niveau social, que culturel ou scolaire. Les enseignants comptent sur une organisation coopérative du travail pour diversifier les formes de rapport au savoir et les modes d'aide à la difficulté. Les enseignants du lycée $\mathrm{C}$ témoignent de postures de retrait chez beaucoup de leurs élèves. Ils se sont rendu compte que les formes pédagogiques qu'ils utilisaient ne répondaient ni aux besoins des adolescents accueillis, ni au nécessaire plaisir qu'un enseignant prend au cours de son activité professionnelle.

«Je m'apercevais, parce que la pédagogie et la didactique qu'on m'avait apprises fonctionnaient moins bien, que les élèves étaient peu réactifs, que moi je m'ennuyais et que j'avais l'impression d'avancer en expérience mais de toucher de moins en moins d'élèves. Etrangement, alors que j'avais plus d'ancienneté, je trouvais que mon enseignement était moins efficace et j'avais l'impression d'être moins utile à mes élèves. Donc ça faisait moins de sens à mon travail.» (Extrait d'un entretien d'une enseignante de mathématiques exerçant au lycée $C$ )

10 Les classes coopératives développées au sein de chaque établissement prennent des formes diverses. Elles ont en commun d'organiser une partie de l'activité des élèves avec du travail en groupe pour susciter du questionnement et de la mutualisation d'idées, de l'aide, de l'entraide et du tutorat au moment des entrainements, pour que l'enseignant ne représente plus la seule ressource humaine capable de répondre aux demandes de déblocages. Il s'agit également de renvoyer des signes aux élèves valorisant leurs efforts, en les incitant à aider des camarades qui viennent les solliciter. Les enseignants activent ainsi un principe de réciprocité entre tuteurs et tutorés (Heber-Suffrin et al., 2011), pour éviter que des inégalités augmentent entre ceux qui aident (et qui en profitent beaucoup) 
et ceux qui se font aider. Toutefois, au sein de chaque établissement, et au sein de chaque cours, la coopération prend systématiquement des formes inédites, en raison de l'absence de méthodes dans le champ de pédagogies de la coopération, d'élèves et de groupes d'élèves différents, d'enseignants aux personnalités et expériences diverses, de contenus disciplinaires spécifiques. Nous avons donc observé autant d'écarts entre deux classes coopératives d'établissements différents qu'entre deux cours au sein d'une même classe.

11 Les entretiens avec les élèves ont eu une durée moyenne de 30 minutes chacun. Les élèves ont été choisis sur la base du volontariat, avec le souci d'un équilibre entre les filles et les garçons. Une fois la démarche expliquée, les élèves intéressés levaient le doigt et le choix s'est d'abord porté sur les premiers qui se sont manifestés. Avant cela, le cadre des entretiens a été présenté devant l'ensemble de chaque classe, avec cet élément de langage :

«Je suis intéressé pour avoir votre avis au sujet de la façon dont vous travaillez dans les classes coopératives. Ce que vous pensez m'intéresse pour comprendre un fonctionnement que vous connaissez bien mieux que moi. Il n'y a pas de bonnes ou mauvaises réponses. Je n'utiliserai pas vos prénoms par la suite.»

Dans chaque établissement, nous avons également sollicité l'avis des enseignants pour interroger des élèves identifiés comme fragiles scolairement, qui ne s'étaient pas portés volontaires au départ, mais qui, en les invitant, acceptaient de répondre à nos questions. Les entretiens se sont déroulés dans des espaces prés de leurs salles de classes, pendant des heures d'enseignement. Ils ont été guidés par les repères suivants: présentation personnelle, avis sur la classe coopérative, le travail autocorrectif, le tutorat en tant que tutoré et en tant que tuteur, la formation à la coopération, l'évaluation éducative, la constitution des groupes, les objectifs du travail en groupe, la notation du travail en groupe, les plans de travail, les conseils coopératifs et d'autres avis. Toutes les déclinaisons de la coopération entre élèves n'ont pas été explorées, l'étude s'est centrée sur le travail en groupe, l'aide, le tutorat et les conseils coopératifs. Chaque entretien a été enregistré, puis entièrement retranscrit.

13 Pour traiter les données, nous avons réalisé une analyse de contenu catégorielle ${ }^{1}$ (Bardin, 1997). Elle est définie comme une «méthode de classification ou de codification, dans diverses catégories des éléments du document analysé pour mieux en faire ressortir les différentes caractéristiques en vue d'en mieux comprendre le sens exact et précis.» (L'écuyer, in Deslauriers, 1988, p. 50). Nous n'avons pas déterminé d'hypothèse exploratoire préalable. Aussi, les conclusions de ce travail prennent la forme d'une synthèse de la palette des avis collectés.

14 Une fois chaque entretien retranscrit et analysé, nous avons réalisé cette synthèse en croisant les idées énoncées individuellement, sans n'en rejeter aucune. La prise en compte des derniers entretiens a révélé de nombreuses redites, signes d'une globale exhaustivité des idées présentées.

\section{Analyse globale des entretiens d'élèves}

\section{Avis généraux sur la classe coopérative}

Les classes coopératives ont été créées par recherche de meilleurs résultats sur le collège. (BA 7'02) « Trouver une nouvelle façon d'apprendre, ils ont voulu tester quelque chose de nouveau et voir si ça nous plaisait. » (BA 7'02) Les classes coopératives proposent des 
méthodes d'enseignement différentes : les conseils de coopération, le travail coopératif, des évaluations par ceintures, des correspondants. (BC 1'41)

Dans un collège, comme de nombreux parents voulaient que leur enfant intègre une classe coopérative, un tirage au sort a été effectué. (BA 2'01) Au début, les autres élèves pensaient que la $6^{\text {ème }}$ coopérative était réservée aux élèves en difficultés. (BA 2'01) Ces moqueries ont ensuite disparu. (BD 18'43)

Les classes coopératives ne diffèrent que légèrement avec les autres classes. De spécifique, les élèves disposent de tutorat et de travail en groupe (CB 12'36). Les élèves possèdent une salle de classe. Ce sont les enseignants qui se déplacent. (BA 1'05)

\section{Responsabilisation}

Les élèves se sentent responsabilisés par l'organisation de la classe : ils travaillent pour leur avenir. (AC 7’42) «On vient pas en cours parce que c'est maman qui amène. On sait qu'on vient pour construire notre avenir. » (AC 7’42) La classe coopérative est un lieu d'efforts, ce n'est pas fait pour s'amuser même si le travail se fait sous des formes différentes. ( $\mathrm{CD}$ 9'32) Elle ne règle pas tous les problèmes mais permet aux élèves d'être plus épanouis. (CD 8'28)

\section{Autonomie}

19 La coopération sert à devenir autonome pour les années de collège suivantes. (AA 10'16) «Pendant la cinquième il n'y aura pas le plan de travail et ils seront pas comme ça les professeurs.» (AA 10'31) Au fil de l'année, les élèves deviennent autonomes et s'appliquent mieux. (AL 1'08) Etre autonome, c'est ne pas déranger la classe, se déplacer pour aider des camarades, leur expliquer le travail, essayer que l'autre comprenne. (AL 10'19)

\section{Coopération}

Le projet de classe coopérative consiste à ce que les élèves s'entraident pour mieux réussir. (CC 0'32) En coopérant, les élèves sont meilleurs que seuls : il y a plus d'idées et le travail est intéressant. (CA 16'16) Travailler en coopérant conduit des élèves à ne pas être en concurrence avec d'autres (CB 13'25) et à avancer pour eux-mêmes. (CB 13'44)

21 Les élèves sont disposés en ilots pour qu'ils puissent coopérer. (BA 5'02, BE 1'20) Pouvoir s'entraider en classe peut être à la fois utile et dérangeant. ( $\left.\mathrm{BC} 6^{\prime} 16\right)$ Les élèves des classes coopératives disposent d'heures spécifiques pour des travaux coopératifs. Ils en profitent pour réaliser leurs devoirs, ce qui réduit leur travail à la maison. (BE 0’47)

En classe coopérative, les élèves comprennent mieux parce que des explications sont possibles. (AD 9'07) «Ça prend le temps de m'expliquer.» ( $\mathrm{AD}$ 9'18) Chacun peut demander de l'aide. (AI 01'45) La classe est coopérative parce que, en cas de besoin, quelqu'un vient aider. (AE 2'38) Elle leur permet de ne pas se retrouver seuls en cas de problèmes (AH 15'31) «Si quelqu'un n'arrive pas, quelqu'un vient nous aider, genre on s'aide entre nous.» (AE 2'38) Dans d'autres collèges non coopératifs, le travail est individuel : l'entraide n'est pas possible et, en cas de difficulté, les élèves sont perdus. (AD 7'53) « Là-bas c'est personnel et ici c'est coopératif. » (AD 7'53) « C'est comme ça, tu es au milieu de ta classe tu comprends rien et après tu es perdu dans le travail et ça t'aide pas. » (AD, 8'16) 
23 Au lycée, des élèves expliquent que coopérer peut conduire à être dépendant de l'aide. C'est pourquoi il est nécessaire de s'être préparé à ne pas pouvoir toujours compter sur les autres. (CA 17'35) La coopération n'aide pas pour l'âge adulte mais surtout pour la scolarité à venir : les acquis sont meilleurs et les habitudes de demander de l'aide en cas de besoin installées. (CC 27’34)

\section{Climat scolaire}

24 Les échanges doivent se faire dans le calme. (AE 5'14) Lors des travaux en groupe, il y a plus de bruit qu'au moment des plans de travail où les élèves réfléchissent seuls le plus souvent. (BE 15’04) La classe coopérative permet d'avoir une complicité avec les enseignants, ce qui est apprécié par les élèves interrogés. Les élèves ont l'impression que les professeurs essaient de les comprendre. (CD 7'16) Le lien est plus simple avec les enseignants ce qui facilite les demandes d'aide. (CD 38'51) «Je me sens plus libre d'aller voir par exemple ma prof principale. L'année dernière mon prof principal j'allais jamais le voir si j'avais des problèmes alors que là j'trouve on a plus confiance, elle nous connait vraiment. » (CD 38'51) S'instaure de la confiance entre les élèves et entre les enseignants et les élèves. (CD 38'36)

\section{Le tutorat en tant que tutoré}

$25 \mathrm{Au}$ début, les élèves essaient par eux-mêmes. ( $\mathrm{AD}$ 1'17, $\mathrm{AK}$ 11'07) Quand un élève rencontre des difficultés ou en ressent le besoin, il peut demander de l'aide. (BA 5'34, BB 2'19, BE 5'32, CA 0'42) Etre aidé n'est pas « rester tranquille. » (AH 6'33) Les tuteurs ne doivent pas intervenir avant que leurs camarades aient essayé de comprendre. (CD 20'14)

Ce sont les enseignants qui indiquent quels sont les moments où l'aide est possible. (AE 2'51, CC 0'32, CD 1'18) Un élève peut être aidé soit par un camarade soit par un enseignant. (AI 02'28, BE 5'23) Si un tuteur ne parvient pas à aider, l'élève s'adresse à l'enseignant. (AJ 2'51, AJ 10'16) S'il obtient une réponse auprès du camarade, cela devient inutile de solliciter un adulte. (AJ 10'05)

Ce sont les élèves qui ont besoin d'aide qui font la démarche de solliciter les tuteurs. (CD 17’04) Comme les élèves sont répartis par ilots, c'est d'abord au sein de ces cellules que les aides sont demandées. (BA 5'02) « C'est plus simple de leur demander à eux, que se lever et demander à d'autres personnes alors que dans notre ilot ils peuvent très bien savoir. " (BA 5’02)

Les aides demandées aux camarades concernent des petits éléments. Cela permet de décharger l'enseignant qui est beaucoup sollicité. (BB 2'59) «Il y aurait tellement de monde à côté de l'enseignant parce que des fois c'est que un tout petit truc qu'on a mal compris et quelqu'un peut nous aider. » (BB 2'59) Le travail coopératif permet de libérer du temps pour que le professeur puisse s'occuper des élèves les plus en difficulté. Il permet également aux élèves de ne pas avoir à attendre que l'enseignant soit disponible. (AJ 3'21)

\section{Aide et travail personnel}

On sollicite un camarade, en priorité un qui fait le même exercice (AL 5'18) ou dont le nom est indiqué sur un tableau d'aide. (AF 4'16, AK 05’37) « Par exemple, si on veut nous 
aider à une leçon ou juste avant une évaluation, on demande et il nous dit oui. » (AD 1'17) Dans certaines classes, la démarche est différente : il s'agit de s'inscrire sur un tableau en y marquant son prénom et son besoin, puis d'attendre que quelqu'un se déplace. (AG 12'35).

Comme les enseignants ne contrôlent pas tout ce que font les élèves, il est possible de faire réaliser son travail par d'autres, mais ça aurait pour conséquence de ne rien apprendre et d'échouer aux évaluations. (AA 7'24, AF 9'46) « Après je saurai rien. Après c'est lui qui va connaitre tout et moi je serai j'aurais rien dans la tête et je connaitrai pas la leçon et pendant les évaluations j'aurai jaune ou rouge. » (AA 8'03) C'est une pratique assimilée par les élèves à du mensonge et à de la triche. (AK 08'37, AL 7'01) Tricher, c'est risquer de décevoir les enseignants et se décevoir soi-même. (AK 09'03) « On sait bien que la personne qui va nous aider n'est pas là pour nous donner des réponses à notre travail. » (AL 7'16) De plus, certains redoutent que les enseignants se rendent compte de cette stratégie de contournement. (AE 11'57)

\section{Demande d'aide et honte}

Demander de l'aide n'est pas évident, surtout en début d'année, parce que les élèves ne se connaissent pas. Mais après un mois de fonctionnement coopératif, le sentiment de honte s'atténue parce que demander montre que l'on cherche à progresser. (BB 6' $\left.52, \mathrm{AJ} 5^{\prime} 40\right) \mathrm{La}$ plupart des élèves de la classe demandent de l'aide facilement, sans aucune honte. (BB 7'30) «J'ai pas particulièrement vu des gens qui n'avaient vraiment pas envie d'avoir de l'aide.» (BB 7'30) Les tutorés ressentent moins de honte à demander de l'aide à des camarades (BD 6'01), en particulier ceux avec qui ils s'entendent bien. (BE 10'10)

Cela ne fait pas honte de demander de l'aide parce que cela permet de s'améliorer. (AG 13'30) «J'ai pas honte, y a plusieurs personnes qui le font dans la classe. Moi aussi je le fais et j'ai pas du tout honte. Parce que sans leur aide moi dès que je serai grand je comprendrai rien. » (AG 13'44) Il n'y a pas de raison à avoir honte dans le sens où tout le monde peut avoir besoin d'aide. (AK 10'24) «Peut-être que le voisin à qui je vais demander lui aussi il ne sait pas la réponse donc il n'y a pas besoin d'avoir honte. » (AK 10'24)

\section{Tétra-aides $^{2}$}

Dans certaines classes, un tétra-aide permet l'organisation de l'aide. (AB 2'24) Vert lorsque tout va bien, jaune pour une question non-urgente, rouge pour une question urgente et bleu quand on est aidé. (AA 1'30) « On est plein dans la classe, il y en a un qui y arrive pas ben la prof elle va l'aider et après si un autre il lui demande ben ils sont occupés, et après pendant ce temps nous soit on met notre tétra-aide en attente soit on va s'écrire dans le tableau et on prend une autre fiche. » (AF 12'00)

\section{Passeports}

Une alternative aux tétra-aides sont les passeports: lorsqu'un élève rencontre une difficulté, il va le déposer près d'un camarade, pour exprimer sa demande. ( $\mathrm{BC}$ 3'37, $\mathrm{BE}$ 5'49) Pour un tuteur, le côté vert du passeport signifie que l'on est disponible pour aider, le rouge indique le contraire. (BD 3'12) 


\section{Etre aidé par un tuteur ou par un enseignant} nécessaire. (AG 12'17) «Oui ça m'est arrivé. Par exemple en espagnol pour les verbes j'avais pas très bien compris et une personne m'a aidé. » (AG 12'17) Tous les élèves, même ceux qui réussissent, rencontrent des difficultés. (AH 7'10). «C'est tout le monde, tout le monde à des difficultés. On n'est pas tous intelligents, y' a des difficultés. » (AH 7’10)

\section{Le tutorat en tant que tuteur}

Un tuteur ne transmet généralement pas de savoirs. (AF 14'11) Avec le tutorat, il n'y a pas d'échange d'idées, contrairement au travail en groupe. (AH 8'55) Le tutorat se passe au sein des classes, pas d'une classe à l'autre. (BC 7'12)

41 Le tuteur est un élève qui va aider un camarade ayant exprimé un besoin d'aide. (AF 4'16) Pour aider, il est nécessaire d'avoir terminé son travail et d'être volontaire. C'est pour cela que certains élèves ne peuvent pas aider. (CD 5’56, CD 16’54) Des élèves faibles sont 
tuteurs. (CD 15'00) Les résultats scolaires ne sont pas un prérequis à cette fonction. (CD 14'18) Cependant, pour aider quelqu'un, il s'agit d'avoir compris les notions que l'on explique. (CA 6'16) «Parce que moi si je comprends pas je pourrai pas leur expliquer.» (CA 6'16) Environ la moitié des élèves de la classe sont des tuteurs. Les autres ne le sont pas à cause d'un manque de confiance en eux-mêmes. (BE 9'08) « Moi, je suis pas super bon dans les matières donc j'ai pas envie de dire importe quoi aux autres. » (BE 9'08)

Dans un établissement, le nombre de tuteurs en activité est limité, pour éviter trop d'agitation dans les classes. (CD 16'25) Ils peuvent refuser une demande d'aide et, s'ils ne savent pas répondre, l'enseignant est alors disponible pour intervenir. (CB 4'07)

Cela ne dérange pas d'aider parce que les élèves ont la possibilité d'indiquer avec leur passeport si cela ne les dérange pas dans leur travail. (BE 11'07) «Quand je suis sur un travail, je me mets en rouge au moins ils viennent pas me demander si je suis libre ou pas. » (BE 11'07) Les élèves qui laissent leur passeport en rouge sont rarement sollicités pour apporter leur aide. ( $\left.B C 5^{\prime} 11\right) C^{\prime}$ 'est utile pour les élèves qui préfèrent travailler seuls. (BC 5'25)

\section{Stratégies d'aide}

Les aides concernent surtout les consignes. (AL 7'34) En effet, quelques élèves demandent de l'aide sans les avoir lues. (AF 13'49) « Parce que parfois ils demandent de l'aide alors qu'ils ont même pas encore lu la consigne. » (AF 13'49) Un tuteur sait que son explication a fonctionné lorsque le tutoré réussit son travail. (AL 11'31)

Le temps d'aide maximum est de 10 minutes. (AG 12'06, CD 17'26) « C'est pas une perte de temps parce que si ils ont pas compris ils ont le droit de pas comprendre comme tout le monde. » (AJ 12'04) De plus, aider des camarades permet au tuteur de mieux comprendre ce qu'il sait. (CA 3'21) «Je vais leur expliquer la chose et vu que moi je la connais un peu je vais leur expliquer encore et ça va un peu plus me rentrer dans la tête.» (CA 3'21) Les deux élèves sortent gagnant-gagnant. (CB 0'33)

Les tuteurs sont des élèves qui ont bien compris comment aider, notamment en ne donnant pas la réponse immédiatement, en encourageant celui qui cherche. (BB 10'03) «Ils savent aussi surtout ce qu'il ne faut pas faire. » (BB 10'26) Pour aider sans donner la réponse, les élèves disent expliquer la consigne, relire les informations, fournir quelques exemples, donner des indices, utiliser des mots qu'il connait. (AB 6'41, AE 8'31, AD 2'30, AF 10'06, AH 4'51) Pour aider, il est possible d'utiliser les ressources présentes dans la classe, notamment les leçons passées. (AG 9'58, AI 3'41) Le tuteur peut également faire l'exercice avec le tutoré et lui poser des questions pour l'aider à comprendre. (AJ 4'37)

Etre tuteur est une tâche délicate parce que ce n'est pas facile d'aider quelqu'un. (BD 7'12) Un tuteur qui ne sait pas répondre à une question oriente l'aide vers quelqu'un d'autre (BD 9'35), notamment un enseignant. (BE 7'05) Le principe de l'aide est de fournir des explications sans empêcher celui que l'on aide à réaliser le travail par lui-même. (BE 8'03) "Je leur explique sans leur donner les réponses pour qu'ils le fassent d'eux-mêmes, pour pas que je dise là c'est ça, là c'est ça. » (BE 8'03) Suite à la formation, une affiche présente dans les classes indique ce que doit faire un tuteur. (AF 7'08) 


\section{Ne pas donner la réponse}

Donner la réponse ou faire le travail à la place de l'autre ne sont pas des stratégies efficaces parce que cela empêche d'apprendre et donc de réussir l'évaluation qui suit. (AB 7'02, AE 9'33, AI 7'00, BD 6'42, CD 12'59) « S'il y a un exercice et le prochain ça sera une évaluation et ben s'il fait l'exercice je lui donne la réponse il pourra pas apprendre et à l'évaluation il saura pas quoi faire.» (AB 7'02) Pour aider, on ne fait pas à la place des autres. Sinon, ce serait trop facile, ça ne servirait pas et personne n'apprendrait. (AD 5'36) Il y a une différence entre donner les réponses et aider. (AK 06'29, 06’34, 06'40) Si le tuteur donne la réponse, c'est lui qui travaille, pas celui qui a demandé l'aide. (BD 6'34, AK 07'28) "C'est comme si c'était ton esclave, comme si tu lui disais prépare moi. » (AK 7’31) Aider, c'est laisser les élèves faire le travail. (AK 6'47)

Donner la réponse n'aide pas parce que ça évite de faire des erreurs, ce qui est nécessaire pour apprendre. (AL 7'52) « Si on a la réponse, on peut pas faire des erreurs et si on fait pas des erreurs, on peut pas apprendre.» (AL 7'52) Se tromper aide à corriger ses erreurs et donc aide à apprendre. (AL 8'23) «À chaque fois que je me trompe je corrige mes erreurs et j'apprends quelque chose.» (AL 8'23) Si le tuteur donne directement la réponse, c'est lui qui apprend, pas celui qu'il aide. (AH 4'19).

\section{Intérêts pour le tuteur}

C'est mieux d'aider les autres pour que tous progressent. (CD 18'13) Cela semble important pour favoriser la réussite des camarades. (CD 19'22) «Si on s'est proposé comme tuteur c'est que ça nous dérangeait pas de pendant 5 minutes mettre notre travail entre parenthèse et aider les autres. C'est bien de ne pas toujours être égoïste. » (CD 19'30) Cet esprit est le plus important en classe coopérative. (CD 18'21) Même lorsqu'aider n'apporte rien, c'est normal de répondre aux demandes d'un camarade parce que tout le monde peut être dans cette situation. (BB 9'24)

51 De plus, aider quelqu'un permet de renforcer ses propres apprentissages parce que l'on découvre de nouvelles façons de s'y prendre. (BB 9'00) « On se rend compte qu'il y avait une solution beaucoup plus simple à l'exercice.» (BB 9'00) Un tuteur consolide ses compétences parce qu'il est amené à les utiliser. (BD 8'52) « Ça peut nous re-entrainer, comme ça on est sûr qu'on sait bien le faire. » (BD 8'52)

Aider provoque également du plaisir, de la satisfaction et du bienêtre parce que cela rend service à quelqu'un. (AE 7'05, AF 15'53, AK 12'20, AL 9'27) «Quand j'aide quelqu'un je me sens mieux, je sens que j'ai fait quelque chose de bien pour les autres et c'est ce que j'aime. » (AL 9'35) «C'est bien pour moi quand j'aide les gens parce que c'est agréable.» (AE 7'05) « Ça fait du bien. Je suis content pour lui.» (AF 16’00) C'est agréable d'aider des camarades à comprendre. (BB 8'14)

Le tutorat a aussi des effets sur la bonne entente entre les élèves: connaissances mutuelles, meilleure ambiance. (CB 1'35): il conduit à rencontrer de nouveaux camarades. Ils se découvrent ainsi des points communs. (CB 2'17) 


\section{La formation à la coopération}

\section{Une formation initiale}

Pour préparer les élèves à cette fonction, une formation à la coopération est réalisée en début d'année. (CA 0'42, CB 6'57, CC 8'19) Ce sont des enseignants qui expliquent les gestes coopératifs. ( $\left.\mathrm{AD} 2{ }^{\prime} 42, \mathrm{AD} 4{ }^{\prime} 37\right)$ Ces gestes ont été étudiés à partir d'exercices de simulation : ces jeux de rôles sont utilisés. (AG 8'18, AF 8'00) «Au début de l'année on a fait une sorte de théâtre et en fait c'est comme si j'étais là et après j'appelle quelqu'un et après lui il me donne les réponses et après au tableau on écrivait non il faut pas donner les réponses. » ( $\left.\mathrm{AF} 8^{\prime} 00\right)$

Cette formation à la coopération est utile parce que, pour les élèves, ce sont des façons de travailler différentes de l'école primaire. ( $\mathrm{AE}$ 12'35) Elle sert à expliquer le fonctionnement, à comprendre les règles et les objectifs. (AG 9'34, CA 4'57) Les tutorés comprennent comment s'organise le tutorat. ( $\mathrm{CB}$ 8'24) Les tuteurs sont formés pour débloquer, ne donner que des indices, pas les réponses, par exemple relire la leçon, se rappeler ce qui avait été dit, ... (CB 3'38, CB 8'11) La formation des élèves au tutorat sert également pour les situations de travail en groupe. (AG 7'22)

\section{La constitution des groupes}

Le premier problème avec le travail en groupe est que certains élèves ne sont pas motivés par l'activité et ils perturbent la concentration. (BB 12'50) Des élèves qui ne s'apprécient pas doivent travailler ensemble. (BC 8'09) En revanche, il est agréable avec des élèves qui sont intéressés. (BB 13'11)

Certains disent préférer se retrouver avec des amis mais reconnaissent que ce serait tentant de bavarder avec eux. (BC $8^{\prime} 57$ ) De plus, si on laissait les élèves créer eux-mêmes ces groupes, certains se retrouveraient seuls. (AH 10'54)

C'est pour cela que ce sont les enseignants qui constituent les groupes. (AE 18'17, $\mathrm{AH}$ 10'11) Ils sont composés de 3, 4 ou 5 personnes. (AG 3'12, AI 10'50, AJ 16'17) Lorsque l'activité n'est pas importante, ce sont les élèves qui se regroupent par affinités. (AG 3'39)

Le second problème est que les élèves qui ne sont pas satisfaits par les groupes ne travaillent pas. (AH 10'25) «Je suis dégouté j'ai pas envie d'être avec eux je préfèrerais être avec d'autres mais du coup ça ne motive pas à fond. » (CA 11'09)

Plusieurs trouvent dommage de ne pas être avec ses copains mais cela permet de travailler avec de nouveaux élèves. ( $\left.\mathrm{AE} 18^{\prime} 46\right)$ « Ça sert à mieux travailler ensemble parce qu'on n'a pas toujours l'habitude de travailler avec les mêmes personnes. » (AE 19'22)

\section{Tirages au sort}

61 Dans certaines classes, les groupes sont constitués au hasard, avec des cartes ou des étiquettes. Les 4 mêmes cartes forment un groupe. (CC 19'49, CD 28'03) Les enseignants s'autorisent à modifier une constitution aléatoire qui poserait des problèmes. (CD 29'23) La constitution des groupes au hasard est intéressante pour éviter de se retrouver avec des amis. (CC 21'29) 
62 En organisant un tirage au sort, on ne sait pas qui constitue les groupes. Les élèves se plaignent moins. (AE 20'32, CD 28'49) «C'est ni la faute des élèves ni la faute des profs c'est le hasard. » La décision ne vient pas de l'enseignant. (CD 29'13) «Les cartes j'trouve c'est un bon système parce que on n'a pas le choix. » (CD 29'13, AE 20'55) Le tirage au sort permet d'éviter de faire des jaloux, de créer des conflits et de perdre du temps à constituer des groupes. (BB 14'30) Si les enseignants constituaient les ilots, cela créerait des conflits entre des élèves qui ne veulent pas se regrouper. (BB 14'46)

\section{Les objectifs du travail en groupe}

63 C'est le cœur du projet de classe coopérative que de travailler en groupe. Dans d'autres classes, les élèves sont plus souvent seuls. (BE 13'04) Les élèves travaillent environ $30 \%$ du temps en groupe. (BB 15'58) Une règle est de chuchoter pour ne pas déranger les autres. (AI 10’07)

\section{Diversifier les façons d'apprendre}

Le travail en groupe est associé au travail individuel pour diversifier les occasions d'apprendre. (AG 5'02) « Dès qu'on va l'avoir fait en groupe après on va le refaire tout seul pour voir si on l'a mieux fait ou pas, si on comprend mieux en groupe.» (AG 5'02) Les élèves disent travailler plus vite en groupe que seuls, parce que les idées fusent. (AI 10'26, AK 03'24)

65 Le travail en groupe diversifie les modes d'apprentissage et fait appel à des formes d'intelligences différentes. (CD 25'01) «Parce que y'a des personnes qui sont intrapersonnelles et qui donc ont besoin de parler, d'avoir les idées de plusieurs personnes.» (CD 25'01) Il aide les élèves qui ne comprennent pas à s'engager autrement dans le travail. (CD 25’41)

\section{Confronter des idées}

Le travail en groupe consiste à résoudre des problèmes communs, difficiles à résoudre seul. (AH 8'31, AE 21'38) La synthèse des idées de chacun favorise l'apprentissage de l'ensemble des élèves. (BB 16'10) « C'est quand on a des grosses activités sur lesquelles on n'a jamais travaillé et du coup on coordonne beaucoup pour vraiment réussir à venir à bout parce une personne n'y arriverait pas. Et du coup avec tout ce que chacun sait ça permet de trouver une solution. » (BB 16'10) Le travail en groupe consiste à échanger des avis susceptibles d'être différents. (BC 11'14) Il sert à ce que les élèves confrontent leurs idées. (CB 11'29) « Dans le travail en groupe, toutes les idées qu'on a, on les met ensemble et là, on voit laquelle convient le mieux, et en fait, on a des raisons pour que l'autre soit mieux que elle, et en fait c'est comme ça qu'on progresse. » (CC 12'55)

Ainsi, les élèves s'entraident et se concertent. (AF 18'12, AJ 1'14) Ils essaient ensuite de se mettre d'accord sur ce que pense chacun. (AK 02'25) «Pour se mettre d'accord, par exemple moi je dis ça et il y a le voisin qui me dit non je trouve ça pas bien et on se met d'accord. » (AK 2'25) Des désaccords naissent et se traitent par des discussions. (AJ 18'27) Tout le monde peut s'exprimer, par l'intermédiaire de «mini-débats. » L'avis des élèves est ensuite argumenté. (CD 24'16) Les oppositions conduisent à chercher des preuves en faisant des recherches. (AK 03'14) Lorsque des avis sont différents au sein d'un groupe, les élèves fournissent leurs arguments et des changements d'avis se produisent. (BD 13'23, CD 
24'39) «Tout le monde met ses réponses en commun et donc du coup des fois ça fait changer d'avis aux gens.» (CD 24'39) «Des fois, tu penses avoir raison puis après tu te rends compte, quand l'autre il explique, qu'en fait tu t'es trompé. » (BD 13'52)

Si le groupe ne parvient pas à se mettre d'accord, chacun fait un travail personnel et le professeur tranche. (AJ 19'09) C'est pour cela que la confrontation précède un temps de correction par l'enseignant. (AH 9'20)

\section{S'entraider}

Travailler en groupe permet à celui qui n'a pas compris d'obtenir de l'aide de ses camarades. (AG 5'59) « Dès que je comprends pas des trucs, mes camarades qui sont dans le groupe eh ben ils m'aident. » (AG 5'59) Le travail en groupe est une source d'aide pour les élèves qui ne savent pas réaliser seuls une consigne. (BD 12'42) « S'il y a quelque chose qu'on sait pas et ben il y a une autre personne qui le sait. » (BD 12'42) Cette entraide permet de débloquer certains élèves. (BE 11'52, CA 14'13, CB 11'36) « Au moins quand on a un petit trou, qu'on comprend pas, comme on est en groupe, normalement tout le monde va comprendre parce qu'on s'aide. » (BE 11'52)

\section{Etre solidaires}

Le travail en groupe sert ainsi à apprendre la solidarité. (AH 9'41) Il rassure les élèves parce qu'ils ne se sentent pas seuls face aux consignes. (BC 11'48) «Quand on fait un travail en groupe on se sent plus à l'aise parce qu'on s'dit si y'en a un qu'arrive pas à ça y'a l'autre qui pourra nous aider.» (BC 11'48)

\section{S'engager}

71 Les élèves qui ne font pas l'effort de s'engager dans le travail en groupe ne peuvent pas en profiter. (CA 15'05) Pour travailler en groupe, ce serait comme dans la vie, il est nécessaire de s'impliquer. Cet engagement est facilité par la présence de plusieurs personnes disponibles pour aider. (CC 15’58) Le travail uniquement individuel ne répond pas à ce besoin. L'effet de groupe a un impact positif sur certains, et cela les motive à travailler (CD 27'01)

Tous les élèves peuvent s'investir dans le travail en groupe, mais on ne peut pas l'imposer à ceux qui ne le veulent pas. (CC 18'10) Des élèves ne travaillent pas dans les groupes. (CC 15'15) Ce serait intéressant de laisser les élèves démotivés pour travailler en groupe faire le même travail seuls. (CC 16’48)

\section{Conclusion}

Voici donc ce que disent des collégiens et des lycéens inscrits dans des classes que leurs enseignants désignent comme coopératives. D'autres textes témoignent de l'avis d'élèves dans le primaire (par exemple, Connac, 2016). A notre connaissance, c'est la première fois où l'on accède à de tels témoignages de jeunes dans le secondaire.

Il apparait une réalité vécue comme incarnée et appréciée. Ces élèves justifient le choix de leurs enseignants par des propos porteurs de responsabilité, d'autonomie, d'altruisme et de démocratie. La classe coopérative est d'abord présentée comme un espace où agit 
une communauté d'apprentissage, le rapport au savoir étant la principale motivation aux engagements. "Il ne faut pas confondre la pédagogie coopérative avec une sorte d'animation psychologique et basiste des groupes humains, promouvant systématiquement le bavardage en lieu et place de la transmission rigoureuse des savoirs, abdiquant toute exigence au nom de la gentillesse, voire de la compassion. » (Meirieu, in Sumputh, Fourcade, 2013, p. 245)

Ceci dit, ces discours véhiculent une altération de la réalité puisque leur essence est la présentation de subjectivités. Autrement dit, ce qui est exprimé par un élève n'est pas forcément fidèle à ce que pensent tous ses camarades, ce qui est dit par un élève d'un établissement ne peut être élargi à l'ensemble de ceux évoluant en classe coopérative. Intervient également un lemme de l'effet Hawthorne (De Landsheere, 1979), à savoir que ce qu'ils disent est déformé par leur conscience de participer à une recherche. Au centre des attentions manifestées par les chercheurs, les élèves se sentent valorisés et certains auraient tendance à embellir la réalité des choses par fierté de participer à un projet qui suscite de l'intérêt. A l'écoute des propos d'élèves, des enseignants ont témoigné ne pas reconnaitre tous les comportements manifestés en classe. Par exemple, certains élèves expliquant l'importance de ne pas trop se faire aider auraient plusieurs fois été surpris en train de demander à des camarades d'exécuter leur travail à leur place.

Il n'en reste pas moins que ces discours d'élèves vont dans le sens de ce que plusieurs recherches autour des pédagogies de la coopération mettent en avant pour autoriser les progrès de tous. (Connac, 2017 b) Ils expliquent nettement que les formes de coopération dissymétrique (aide et tutorat) ont besoin de réciprocité (Héber-Suffrin et al., 2011) pour que tous les élèves aident et qu'aucun ne se sente dévalorisé. Le statut d'élève tuteur répond à plusieurs critères : avoir reçu une formation préalable (Bensalah, Berzin, 2009), être volontaire, alterner fonction de tuteur et celle de tutoré. Les formes de coopération symétrique (entraide et travail en groupe) revendiquent une constitution aléatoire des groupes, des fonctions de structuration démocratique des échanges, des consignes signifiantes, une alternance entre travail individuel et coopératif, l'entretien du calme (Meirieu, 1996, Barlow, 2002, Reverdy, 2016). Il apparait judicieux d'autoriser des élèves ne souhaitant pas travailler en groupe réaliser seuls le même travail. L'individualisation des apprentissages réclame à la fois un accompagnement de l'autonomie, pour susciter des postures de secondarisation (Bautier, Goigoux, 2004), ainsi que l'organisation d'une structure coopérative des échanges, notamment en lien avec un conseil d'élèves. (Le Gal, 2002)

77 Au regard des objectifs exprimés par les enseignants, l'analyse des propos de leurs élèves font apparaitre un investissement plus grand dans les tâches scolaires, des satisfactions reconnues à aider un ou une camarade, ainsi qu'une meilleure prise en compte, par l'organisation coopérative du travail, des différences interindividuelles présentes au sein d'un groupe. Les classes coopératives en collège et lycée seraient des dispositifs pertinents pour susciter de la référence interne par de la motivation d'innovation (Favre, 2015) et pour répondre aux besoins élémentaires en matière de différenciation pédagogique. (CNESCO, 2017)

Ces témoignages d'élèves ne font qu'éclairer leur réalité scolaire. Bien évidemment, ils ne suffisent pas à justifier la systématique pertinence des classes coopératives dans le secondaire. Notre accompagnement par la recherche de ces équipes a d'ailleurs plusieurs fois souligné, au démarrage de ces projets, des éléments critiques de ce qui était constaté. En pédagogie, rien ne va donc de soi et les facilitateurs se cachent souvent dans des 
détails. La recherche de l'efficacité nécessite de multiples précautions et guides d'analyse. Nous espérons avoir pu faire apparaitre que l'avis des acteurs que sont les élèves pouvait s'avérer pertinent pour l'évaluation d'une partie d'un projet de classe coopérative.

\section{BIBLIOGRAPHIE}

Barlow, M. (2002). Le travail en groupe des élèves. Paris : Bordas.

Bardin, L. (1997). L'analyse de contenu. Paris : PUF.

Bautier, E., Goigoux, R. (2004). Difficultés d'apprentissage, processus de secondarisation et pratiques enseignantes : une hypothèse relationnelle, Revue Française de Pédagogie, 148, 89-100.

Bensalah, L., Berzin, C. (2009). Les bénéfices du tutorat entre enfants, L'orientation scolaire et professionnelle, $\mathrm{n}^{\circ} 38 / 3,325-351$.

CNESCO, (2017). Différenciation pédagogique - Comment adapter l'enseignement pour la réussite de tous les élèves? Paris : CNESCO/IFE.

Connac, S. (2009). Apprendre avec les pédagogies coopératives, démarches et outils pour l'école. Issy-lesMoulineaux : ESF éditeur.

Connac, S. (2016). Autonomie, responsabilité et coopération : ce qu'en disent les élèves utilisant un plan de travail. Éducation et socialisation. En ligne, 4, 2016, URL : http://edso.revues.org/1725

Connac, S. (2017 a). La coopération entre élèves. Futuroscope : Editions Canopé.

Connac, S. (2017 b). Enseigner sans exclure - La pédagogie du colibri. Paris : ESF Editeur.

De Landsheere, G. (1979). Dictionnaire de l'évaluation et de la recherche en éducation. Paris : Presses universitaires de France.

Deslauriers, J.P. et al. (1988). Les méthodes de la recherche qualitative. Sillery : Presses de l'Université du Québec.

Etienne, R. (2009). Accompagner le changement en éducation scolaire, une évolution décisive pour former les enseignants à l'université ? In R. Etienne, M. Altet, C. Lessard, L. Paquay, P. Perrenoud, L'université peut-elle vraiment former les enseignants ? Bruxelles: De Boeck. P. 181-199.

Favre, D. (2015). Cessons de démotiver les élèves. Paris : Dunod.

Heber-Suffrin, C. Renon Thouret, C., Desgroppes, N., Vidricaire, A. (2011). Parier sur la réciprocité, vivre la solidarité. Lyon : Chronique Sociale.

Howden, J. Rouiller, Y. (2009). La pédagogie coopérative. Montréal, Editions Le Chenelière, 2009.

Le Gal, J. (2002) Les droits de l'enfant à l'école, pour une éducation à la citoyenneté. Bruxelles : De Boeck et Belin.

Meirieu, P. (1996). Itinéraire des pédagogies de groupe - Apprendre en groupe. Lyon : Chronique Sociale.

Merleau-Ponty, M. (1945). Phénoménologie de la perception. Paris : Gallimard

Perrenoud, P. (2004). Métier d'élève et sens du travail scolaire. Issy-les-Moulineaux : ESF Editeur. 
Reverdy, C. (2016). La coopération entre élèves : des recherches aux pratiques. Dossier de veille de l'IFÉ no114. Lyon : ENS Lyon.

Sumputh, M., Fourcade, F. (2013). Oser la pédagogie coopérative complexe. Lyon : Chronique Sociale.

Perrenoud, P. (2004). Métier d'élève et sens du travail scolaire. Issy-les-Moulineaux : ESF éditeur.

Veltcheff, C. Vincent, J. F. (2016). Mémento pour la coopération entre élèves au collège et au lycée. Poitiers : Editions Canopé, 2016. En ligne : https://www.reseau-canope.fr/climatscolaire/agir/ ressource/ressourceId/memento-pour-la-cooperation-entre-eleves-au-college-et-au-lycee.html

\section{NOTES}

1. L'analyse de contenu catégorielle expliquée à des étudiants en Sciences de l'Education https:// www.youtube.com/watch?v=MNWq9-IkYvA

2. http://bdemauge.free.fr/tetraaide.pdf

\section{RÉSUMÉS}

Des classes coopératives voient le jour dans des collèges et lycées de l'enseignement public en France. Cette recherche s'intéresse à ce que pensent les élèves inscrits dans ces classes. Elle propose une synthèse d'un corpus de 22 entretiens semi-directifs, réalisés au sein de 11 classes de deux collèges et d'un lycée. Les entretiens mettent à jour l'avis des élèves sur plusieurs points : l'organisation de l'aide et $d u$ tutorat, le travail en groupe, l'individualisation du travail, les conseils coopératifs et, plus largement les classes coopératives qui les accueillent.

Cooperative classes appear in public middle schools and high schools in France. This research deals with the feelings and thoughts of the pupils studying in those classes. It offers an overview of a corpus of 22 semi-guided interviews, done within 11 classes in two middle schools and one high school. The interviews update what pupils think on several subjects: organisation of assistance and mentoring, group work, work personalization, cooperative council and to areat extent the cooperative classes which host them.

\section{INDEX}

Keywords : student council, cooperation, work plan, group work, mentoring

Mots-clés : conseil d'élèves, coopération, plan de travail, travail en groupe, tutorat

\section{AUTEUR}

SYLVAIN CONNAC

LIRDEF, Université Paul Valéry de Montpellier 